\title{
H-mode Access in the low density regime on JET
}

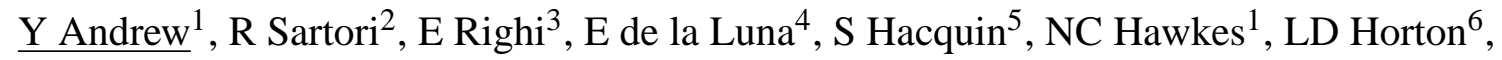
D Howell $^{1}$, A Huber ${ }^{7}$, A Korotkov ${ }^{1}$, MG O’Mullane ${ }^{8}$ and JET-EFDA contributors *

${ }^{1}$ Euratom/UKAEA Fusion Asociation, Culham Science Centre, Abingdon, OX14 3DB, UK

${ }^{2}$ EFDA CSU, c/o MPI fur Plasmaphysik, 2 Boltzmannstrasse, 85748 Garching, Germany

${ }^{3}$ European Commission, Research Directorate General, B-1049 Brussels

${ }^{4}$ Asociasion EURATOM-CIEMAT para Fusion, CIEMAT, Madrid, Spain

${ }^{5}$ CFN-Associacao Euratom/IST, Av. Rovisco Pais, 1049-001 Lisboa, Portugal

${ }^{6}$ Max-Plank-Institut fur Plasmaphysik, EURATOM Association, D-85748, Garching, Germany

${ }^{7}$ Institut fur Plasmaphysik, Forschungszentrum Julich GmbH, EURATOM Association, Trilateral Euregio Cluster, D-52425 Julich, Germany

${ }^{8}$ Department of Physics, University of Strathclyde, Glasgow, UK

1. Introduction In all tokamaks the power threshold for H-mode access, $P_{t h}$, is observed to increase approximately linearly with density [1]. However, a minimum $P_{t h}$ as a function of $n_{e}$ has been demonstrated on several tokamaks including COMPASS-D [2, 3], ALCATOR CMod [4] and DIII-D [5]. The $P_{t h}$ for the ITER ELMy H-mode may exceed the available heating power except at densities appreciably lower than the desired operating condition of $\sim 10^{20} \mathrm{~m}^{-3}$. An operating scenario under consideration for ITER starts with the transition into H-mode at low $n_{e}$ followed by an increase in $n_{e}$ to the required operating condition. Hysteresis between the $\mathrm{L}-\mathrm{H}$ and the $\mathrm{H}-\mathrm{L}$ transitions is expected to maintain the $\mathrm{H}$-mode during the $n_{e}$ rise. Therefore, it is important to characterise $\mathrm{H}$-mode access in present day tokamaks under conditions of very low density.

The departure of $P_{t h}$ on JET from the ITER $n_{e}$ scaling at very low densities was first reported by Horton et al in [6], Righi et al. in [7] and Sartori et al. in [8]. Low density data from COMPASS-D, JT-60U and JET have also been compared with Alfven Drift Instability theory by Igitkhanov et al. in [9]. This earlier JET work is extended here with experimental results from $n_{e}, B_{t}$ and $q_{95}$ scans under conditions of low plasma density both with the Septum and the Septum Replacement Plate (SRP) in the MkII Gas Box (MkIIGB) divertor configurations.

2. Low Density L-H Transition Experiments Density scans were performed with the MKIIGB Septum divertor at $I_{p} / B_{t}$ values of $2.5 \mathrm{MA} / 2.7(2.6) \mathrm{T}, 2.5 \mathrm{MA} / 2.4 \mathrm{~T}$ and 2.2 MA/2.4 T. The L-mode target density was held constant, while the additional heating was slowly ramped up at a rate of $1 \mathrm{MW} / \mathrm{s}$, as illustrated by an example shot in figure 1. The L-H transition for this shot is clearly seen as a sharp drop in the divertor $D_{\alpha}$ signal and corresponds to a reduction in turbulence as measured by reflectometry shown in figure 1(b). Generally, NBI heating was used for the higher density plasmas, but for the lowest densities it was necessary to use ICRH additional heating power only, due to operating constraints. All the plasmas examined used a lower single null configuration, with both inner and outer strike points on the vertical (2004)

${ }^{*}$ See Appendix of J Pamela et al., Fusion Energy 2004, (Proc. 20 ${ }^{\text {th }}$ Int. Conf. Vilamoura, 2004) IAEA Vienna 
(a)

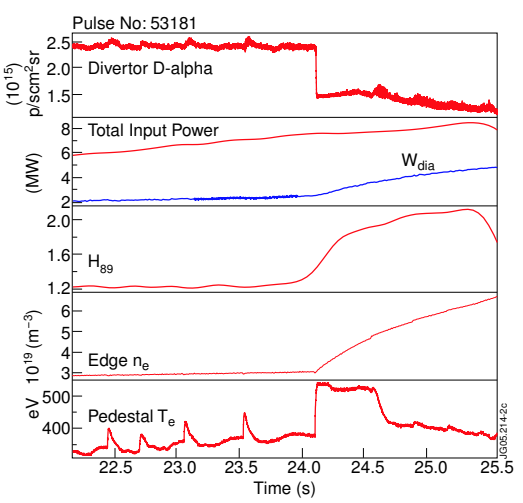

(b)

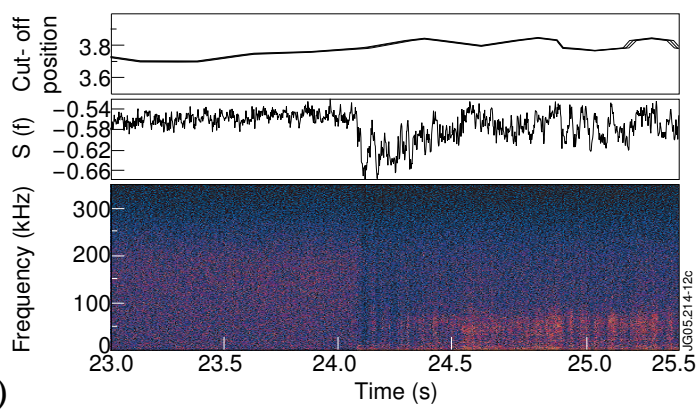

Figure 1: (a)General plasma parameters for an example MkIIGB Septum shot (53181) part of the density scan at 2.5 MA/2.6 T and (b) from top to bottom, reflectometry measurements of radial position of the cut-off layer, integrated spectrogram and a sliding FFT spectrogram of the reflected signal for shot 53181.

targets of the MkIIGB divertor and had deuterium fuelling. The pedestal electron temperature, $T_{e}^{p e d}$, in these shots has been measured with a multichannel ECE radiometer with a time resolution of $1 \mathrm{~ms}$, while the the line average edge $n_{e}$ is the interferometer measured line integrated $\bar{n}_{e}$ divided by the chord length in the plasma.

The values of $P_{t h}$ and $T_{e}^{p e d}$ at the L-H transition for the three sets of $I_{p} / B_{t}$ scans with the MkIIGB Septum divertor are shown in figure 2 as a function of edge density. $P_{t h}$ is defined as the total input power less shine through power losses for NBI, less the rate of change of stored magnetic energy. All three sets of data show a minimum in $P_{t h}$ as a function of density and clearly demonstrate that $P_{t h}$ deviates from the ITER $n_{e}$ scaling for the lowest density plasmas. $T_{e}^{p e d}$ also has a dependence on $n_{e}$ in the low density regime, with the highest $T_{e}$ values in the scans at the lowest values of $n_{e}$, which indicate that $T_{e}$ cannot be the controlling parameter for $\mathrm{H}$-mode access under the low density regime.

The effect of plasma $Z_{\text {eff }}$ on $P_{t h}$ has been investigated by subtracting the bulk radiated power from $P_{t h}$ to give $P_{S E P}$ i.e. the power flowing across the separatrix in to the SOL. $P_{S E P}$ and $Z_{e f f}$ are plotted in the top and bottom graphs respectively, in figure 3 as a function of edge $n_{e}$. The edge $n_{e}$ dependence of $P_{S E P}$ is very similar to that of $P_{t h}$ and $Z_{e f f}$ decreases monotonically with increasing $n_{e}$, showing no evidence of a sharp turning point.

On DIII-D increased values of $P_{t h}$ at low density were attributed to NBI heating required during the initial current ramp up phase to avoid locked modes [5]. When an error field correcting coil was used on DIII-D to reduce the locked mode density limit, the increase in $P_{t h}$ at low density was eliminated. Since it was not necessary on JET to use additional NBI heating during any phase of these ICRH only heated plasmas to avoid locked modes, the higher values of $P_{t h}$ at low $n_{e}$ cannot be attributed to surplus power. However, analysis of the JET low-density shots shows that they are indeed very close to the locked mode density limit at the L-H transition.

It is also interesting to note that $P_{t h}$ is influenced by a change in $I_{p}$ at constant $B_{t}$ at the lowest values of $n_{e}$. In figure 2 at $n_{e}=1.8 \times 10^{19} \mathrm{~m}^{-3}, P_{t h}$ is very similar for $I_{p} / B_{t}=2.5 \mathrm{MA} / 2.4 \mathrm{~T}$ and 2.2 MA/2.4 T. However, at $n_{e}=1.3 \times 10^{19} \mathrm{~m}^{-3}$ and $0.8 \times 10^{19} \mathrm{~m}^{-3}, P_{t h}$ is $1.5-2 \mathrm{MW}$ higher at $I_{p} / B_{t}$ 

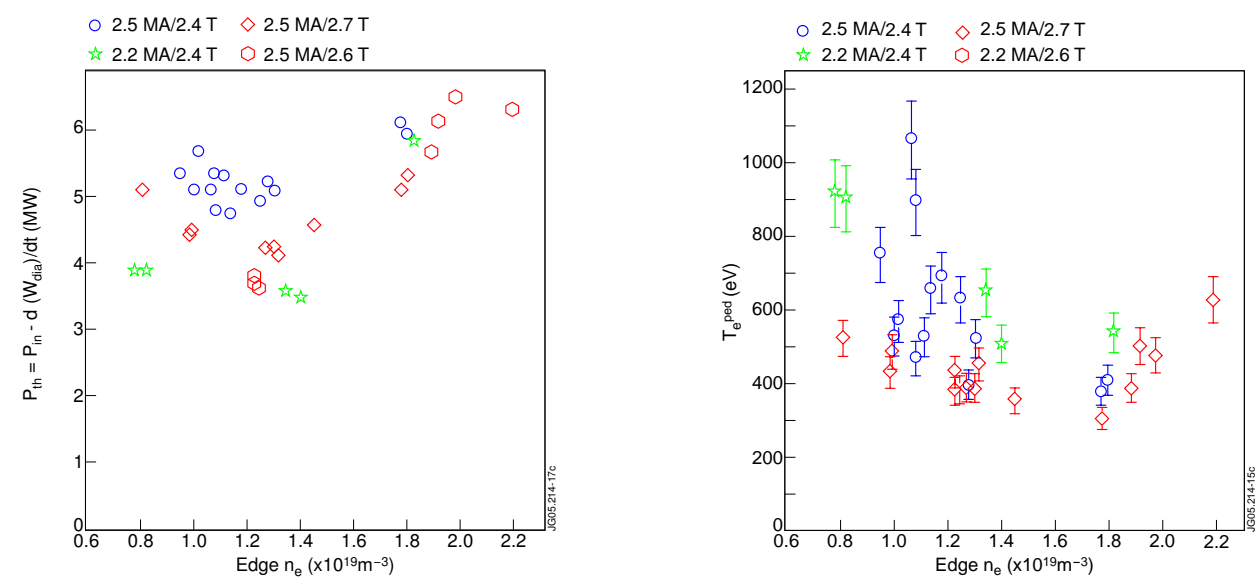

Figure 2: $P_{t h}$ and corresponding pedestal $T_{e}^{\text {ped }}$ values plotted as a function of the edge $n_{e}$ for MkIIGB Septum divertor.

of 2.5 MA/2.4 T than at 2.2 MA/2.4 T. On the other hand, in a separate $I_{p}$ ramp experiment at $B_{t}=2.4 \mathrm{~T}, I_{p}$ was varied from $2.5 \mathrm{MA}$ to $3 \mathrm{MA}$ at the $\mathrm{L}-\mathrm{H}$ transition. The edge edge $n_{e}$ was held constant at $1.3 \times 10^{19} \mathrm{~m}^{-3}$ and $P_{t h}$ demonstrated no dependence on $I_{p}$ in the range scanned. The contrast between these two results may be due to the difference in the $I_{p}$ range explored.

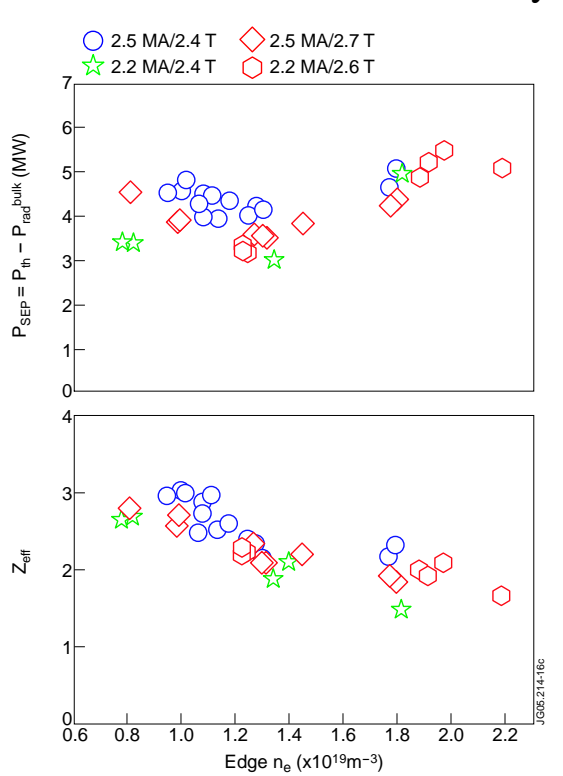

Figure 3: $P_{S E P}$ and $Z_{\text {eff }}$ values plotted as a function of the edge $n_{e}$ for MkIIGB Septum divertor.
The $n_{e}$ scan carried out at 2.5 $\mathrm{MA} / 2.7(2.6) \mathrm{T}$ was repeated with the MKIIGB SRP divertor and the values of $P_{t h}$ and $T_{e}^{\text {ped }}$ from this scan are compared in figure 4(a) and (b). Despite lowering the SRP plasma density to $n_{e}=1.1 \times 10^{19} m^{-3}$, no evidence of a departure of $P_{t h}$ from the ITER scaling is observed. Therefore, if a minimum $P_{t h}$ exists with the SRP it is lower than that with the divertor septum in place $\left(n_{e}=1.2 \times 10^{19} \mathrm{~m}^{-3}\right)$. It is also interesting to note that removal of the septum made little difference to the $T_{e}^{\text {ped }}$.

3. Conclusions Density scans carried out on JET with the MkII GB septum divertor have shown that at very low plasma densities a minimum in $P_{t h}$ exists. The increase in $P_{t h}$ does not appear to be dependent on increasing $Z_{\text {eff }}$ at the lowest densities, with subtraction of the bulk radiated power making no significant difference to the $n_{e}$ dependence. There is a sensitivity of $P_{t h}$ to $q_{95}$ at fixed $B_{t}$ at the lowest densities, with a decrease in $I_{p}$ from 2.5 MA to 2.2. MA reduces $P_{t h}$ by $1.5-2 \mathrm{MW}$. The effect of the divertor septum on H-mode access in the low density regime was examined by repeating reference density scans with the SRP, but no evidence of departure of $P_{t h}$ from the standard ITER scaling was found for lowest densities 

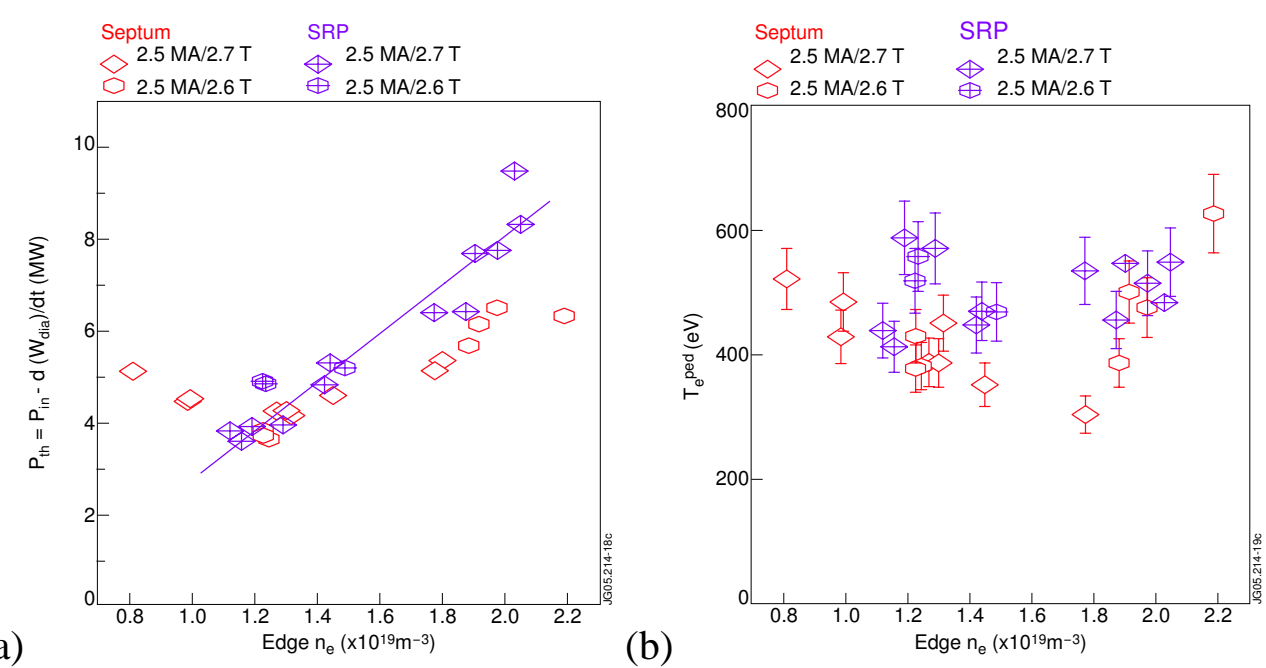

Figure 4: $(a) P_{t h}$ and $(b) T_{e}^{p e d}$ at the L-H transition plotted as a function of edge $n_{e}$ at $2.5 \mathrm{MA} / 2.7$ Tfor the MkIIGB Septum and the MkIIGB SRP divertors

explored. If a minimum $P_{t h}$ exists it has been shifted to an even lower value of $n_{e}$ with the removal of the septum. Finally, $T_{e}^{\text {ped }}$ was found to be very similar for the two sets of divertor data.

\section{Acknowledgement}

This work was funded jointly by the United Kingdom Engineering and Physical Sciences Research Council and by the European Communities under the contract of Association between EURATOM and UKAEA. The views and opinions expressed herein do not necessarily reflect those of the European Commission. This work has been carried out within the framework of the European Fusion Development Agreement. The authors wish to thank R Groebner for valuable discussion and his encouragement in the preparation of this work.

\section{References}

[1] Takizuka T for the ITPA H-mode Power Threshold Database Working Group, Plasma Phys. Control. Fusion 46 (2004) A227- A233.

[2] Fielding SJ, Ashall JD, Carolan PG, Colton A, Gates D, Hugill J, Morris AW, Valovic M, et al., Plasma Phys. Control. Fusion 38 (1996) 1091-1102.

[3] Fielding SJ, Valovic M, Carolan PG, Gates DA, Hunt C, Leahy P, Morris AW et al. Plasma Phys. Control. Fusion 40 (1998) 731-735.

[4] Hubbard AE, Boivin RL, Drake JF, Greenwald M, In Y, Irby JH, Rogers BN, and Snipes JA, Plasma Phys. Control. Fusion 40 (1998) 689-692.

[5] Carlstrom TN and Groebner RJ, Phys. Plasmas 3(5) (May 1996) 1867 -1874.

[6] Horton LD, GD Conway, NC Hawkes, J Lingertat, CG Lowry, CF Maggi, E Righi, G Saibene, R Sartori, B Shunke, ACC Sips, DFH Start, 26th EPS Conference on Contr. Fusion and Plasma Phys., Maastricht, The Netherlands, 14-18 June 1999.

[7] Righi E, Campbell DJ, Conway GD, Hawkes NC, Horton LD, Maggi CF, Saibene G, Sartori G and Zastrow K-D, Plasma Phys. Contol. Fusion 42 (2000) A199-A204.

[8] Sartori R, G Saibene, LD Horton, M Becoulet, R Budney, D Borba, A Chankin, GD Conway, G Cordey, D McDonald, K Guenther, MG von Hellermann, Yu Igithkanov, A Loarte, PJ Lomas, O Pogutse and J Rapp,Plasma Phys. Control. Fusion 46 (2004) 723-750.

[9] Igitkanov Yu, Pogutse O, Connor J, Janeschitz G, Fielding S, Sartori R and Righi EJ, 27th EPS Conference on Contr. Fusion and Plasma Phys., Budapest, 12-16 June 2000 ECA 24B (2000) 11561159. 